Tekiner et al. HCA Healthcare Journal of Medicine (2020) 1:COVID-19

https://doi.org/10.36518/2689-0216.1131

\title{
Education
}

\section{COVID-19 Era Requires Urgent Integration of Virtual Reality Simulations in Medical Education}

Halil Tekiner, PhD, ${ }^{1}$ Cemal Alper Kemaloglu, MD, ${ }^{2}$ Achmet Ali Tsaous, BSc ${ }^{3}$

\section{Abstract}

\section{Description}

The COVID-19 pandemic continues to cause an unprecedented level of disruption across every part of the globe. This disruption detrimentally affects the field of medical education; in particular, anatomical, surgical and clinical training, all which require hands-on experience. On the other hand, the pandemic not only brings new challenges in medical education but also opportunities for testing the validity of our old education paradigms and for developing the ability to embrace a rapid pace of change in better integrating new technologies in the medical curriculum. We contend that the future of medical education lies in the successful use of VR and similar technologies in medical education. Even though this does not mean it can fully replace hands-on training, at present it seems to be the best way possible to support modern medical education in times of limited physical interaction, such as pandemics. However, future studies with robust, comprehensive and culture specific designs are clearly necessary in order to determine the value and feasibility of this approach.

\section{Keywords}

COVID-19; medical education; virtual reality; simulation-based educations; graduate medical education; educational virtual reality; coronavirus infections; SARS-CoV-2; simulation training

Epictetus once said, "The greater the difficulty, the more glory in surmounting it." This uplifting quotation means more than ever these days as the COVID-19 pandemic continues to cause an unprecedented level of disruption across every part of the globe. This disruption detrimentally affects the field of medical education, especially anatomical, surgical and clinical training, which require hands-on experience., ${ }^{1,2}$

This COVID-19 era has pivoted to rely more heavily on distance learning and internet platforms, including live and recorded lectures. While a great deal of effort is made to provide high quality education via these modalities, feedback from both students and faculty members varies greatly. One of the main criticisms is that the current tools used widely in distance learning do not always adequately replace some of the approaches and techniques used during traditional face-to-face education and, as a result, the quality of education suffers. ${ }^{3,4}$
Considering the pandemic is likely to continve into the following semester(s) and that we might experience multiple waves, medical schools will again pause clinical rotations, and traditional face-to-face methods of teaching may remain unavailable to medical students. Therefore, it is crucial that we urgently re-design our educational approaches and environment in order to deal effectively with this new way of teaching. Yet, the only viable option, one that will ensure ongoing education of at least a sufficient quality, is a distance learning model that effectively utilizes recent advancements in internet-based technologies. ${ }^{5,6}$

In this respect, several promising tools have been developed. Of particular interest are those that focus on integrating online learning with practical activities delivered via virtual reality (VR) simulations or even advanced artificial intelligence (AI). ${ }^{7}$ These options could provide powerful learning opportunities for

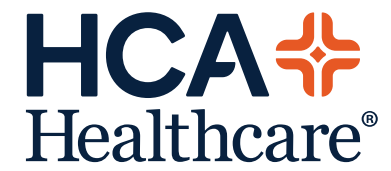

www.hcahealthcarejournal.com

(c) 2020 HCA Physician Services, Inc. d/b/a Emerald Medical Education

\author{
HCA Healthcare \\ Journal of Medicine
}


Table 1. Advantages and disadvantages of using VR simulations in medical education. ${ }^{1,6,12-15}$

\section{Advantages}

- VR technology can successfully mimic different types of medical settings and promote individualized and interprofessional education.

- There are virtually unlimited possibilities for a tailored design, one which appeals to a variety of learning styles.

- It can help to develop students' confidence and enable them to learn at their own pace with increased flexibility, as they can take their classes at any time.

- It is easily accessible from anywhere via smartphones, iPads, and laptops.

- SBE provides students variety of opportunities to practice their skills in a safer environment then in real life.

- It provides valuable practice opportunities for extreme situations or rare events that are difficult to re-create in real settings.

- It can maximize multidisciplinary exchanges to interact and practice necessary skills that ensure a high quality of care.

- It can be utilized in a wide variety of cultural and geographic environments, and the collected can be used to inform further intercultural studies.

\section{Disadvantages}

- VR technology is expensive and needs technicians for its maintenance; however, there is a distinct lack of technological funding support available at many academic institutions.

- It is not accessible for everyone or in those places which have limited internet facilities.

- It may be a difficult tool to use, especially for students who have limited access to technology.

- It may also be challenging for some educators due to the learning curve associated with learning about and using the new technologies.

- A high level of expertise is required to design activities in the platform.

- There is a possible risk for students who learn communication skills exclusively in VR, AR or MR, as they may not be able to cope face-to-face with a real patient.

- Some students might become addicted to the technology and ignore other types of learning.

- Some users might experience adverse effects, like dizziness or nausea, while using the VR headset or other equipment. medical students, who would be able to simulate telemedicine visits with patients. ${ }^{5,8}$ The uses of these virtual or simulated experiences in preparing the next generation for telemedicine range widely, from patient monitoring and healthcare information technology to information analysis collaboration and intelligent diagnosis assistance. ${ }^{9}$

Indeed, VR, augmented reality (AR), binocular omni-orientation monitors (BOOM), mixed reality (MR) and simulation-based education (SBE) seem to be the best possible options for imitating authentic medical settings. ${ }^{5,10}$ All of these have the potential to be successfully integrated within the current medical curricula and provide well-tailored content selection. ${ }^{2}$

Since 21st century-students are likely to prefer a more independent, technology-driven and task-based method of studying, this novel approach is gradually becoming more and more integrated into medical curricula. Its merits are also recognized by the medical education program accreditation standards in the United States and Canada, where it is stated that "if a medical student does not encounter patients with a clinical condition, the medical student should be able to remedy the gap by a simulated experience"." This approach, however, has both advantages and disadvantages that need to be assessed carefully., ${ }^{1,612-15}$ (Table 1)

Recent studies on the use of VR technologies confirm its promising contributions to medical education. Sultan et al. concluded that the use of VR $360^{\circ}$ videos resulted in a significant improvement in students' skills acquisition, knowledge retention and satisfaction levels." The study also concluded that VR is likely to serve as an essential tool in medical education for the present generation." Likewise, Aabersold et al. showed the potential for VR simulations to have a positive impact on nursing student performance. ${ }^{16}$ Furthermore, Dyer et al. claimed that VR immersion training might 
be an effective method for teaching medical students to develop empathy in addition to creating an area for library partnerships. ${ }^{17}$

Despite these positives, we should also note that the current literature on the use of VR technology in medical education has some significant limitations. First, almost all studies were conducted in high-income countries. ${ }^{10}$ Second, the majority of studies evaluate only the usability and acceptability of VR simulation, while few studies have measured the effects of VR simulation on non-technical skills development.12 Third, while VR and other simulated experiences have traditionally been integrated to augment learning, there is insufficient evidence to draw any conclusions regarding their effectiveness when they become the primary modality.

Another problem we should address in this regard is precisely how to establish the most effective way of measuring students' knowledge. The current practice of administering online tests has several pitfalls and security concerns. Instead of using the measuring test results from a scale of 100 , reaching a certain number of correct answers (namely, correct practices in the VR environment) without mistakes could be a more effective and engaging technique for testing. ${ }^{18}$

In brief, the COVID-19 pandemic not only brings new challenges in medical education but also opportunities for testing the validity of our old education paradigms and for developing the ability to embrace a rapid pace of change in better integrating new technologies in the medical curriculum. We contend that the future of medical education lies in the successful use of VR and similar technologies in medical education. Even though these virtual options cannot fully replace hands-on training, it seems to be the best option to support modern medical education in times of limited physical interaction, such as pandemics. However, future studies with robust, comprehensive and culture-specific designs are clearly necessary in order to determine the value and feasibility of this approach.

\section{Conflicts of Interest}

The authors declare they have no conflicts of interest.

\section{Author Affiliations}

1. Department of the History of Medicine and Ethics, Erciyes University School of Medicine, Kayseri, Turkey

2. Department of Plastic Reconstructive and Aesthetic Surgery, Erciyes University School of Medicine, Kayseri, Turkey

3. Anadolu University Faculty of Pharmacy, Eskisehir, Turkey

\section{References}

1. Ferrel MN, Ryan JJ. The Impact of COVID-19 on Medical Education. Cureus. 2020;12(3):e7492. Published 2020 Mar 31 https://doi.org/10.7759/ cureus.7492

2. Goh PS, Sandars J. A vision of the use of technology in medical education after the COVID-19 pandemic. MedEdPublish. 2020;9(1):49. https:// doi.org/10.15694/mep.2020.000049.1

3. Ahmed H, Allaf M, Elghazaly H. COVID-19 and medical education [published correction appears in Lancet Infect Dis. 2020 May;20(5):e79]. Lancet Infect Dis. 2020;20(7):777-778. https://doi. org/10.1016/s1473-3099(20)30226-7

4. Almarzooq ZI, Lopes M, Kochar A. Virtual Learning During the COVID-19 Pandemic: A Disruptive Technology in Graduate Medical Education. J Am Coll Cardiol. 2020;75(20):2635-2638. https://doi. org/10.1016/j.jacc.2020.04.015

5. Tudor Car L, Soong A, Kyaw BM, Chua KL, Low-Beer N, Majeed A. Health professions digital education on clinical practice guidelines: a systematic review by Digital Health Education collaboration. BMC Med. 2019;17(1):139. Published 2019 Jul 18. https://doi.org/10.1186/s12916-0191370-1

6. Rose S. Medical Student Education in the Time of COVID-19. JAMA. 2020;323(21):2131-2132. https://doi.org/10.1001/jama.2020.5227

7. Yoo J, Brownlee EA. The past, present, and future of virtual reality in higher education. In: Okojie MCPO, Boulder TC, eds. Handbook of Research on Adult Learning in Higher Education. Hershey, PA: IGI Global; 2020:633-657.

8. Vozenilek J, Huff JS, Reznek M, Gordon JA. See one, do one, teach one: advanced technology in medical education. Acad Emerg Med. 2004;11(11):1149-1154. https://doi.org/10.1197/j. aem.2004.08.003

9. Pacis DMM, Subido EDC, Bugtai NT. Trends in telemedicine utilizing artificial intelligence. AIP Conference Proceedings. 2018;1933(1):040009. https://doi.org/10.1063/1.5023979

10. Kyaw BM, Saxena N, Posadzki P, et al. Virtual Reality for Health Professions Education: Systematic Review and Meta-Analysis by the Digital Health Education Collaboration. J Med Internet Res. 2019;21(1):e12959. Published 2019 Jan 22. https://doi.org/10.2196/12959 
11. Sultan L, Abuznadah W, Al-Jifree H, Khan MA, Alsaywid B, Ashour F. An Experimental Study On Usefulness Of Virtual Reality $360^{\circ}$ In Undergraduate Medical Education. Adv Med Educ Pract. 2019;10:907-916. Published 2019 Oct 30. https:// doi.org/10.2147/amep.s219344

12. Bracq MS, Michinov E, Jannin P. Virtual Reality Simulation in Nontechnical Skills Training for Healthcare Professionals: A Systematic Review. Simul Healthc. 2019;14(3):188-194. https://doi. org/10.1097/sih.0000000000000347

13. Calin RA. Virtual reality, augmented reality and mixed reality-trends in pedagogy. Social Sciences and Education Research Review. 2018;5(1):169-179.

14. Lilly J, Kaneshiro KN, Misquith C, Dennett B. Creating a new "reality" for medical education: the Nexus Reality Lab for virtual reality. J Med Libr Assoc. 2019;107(4):609-610. https://doi. org/10.5195/jmla.2019.784

15. Uruthiralingam U, Rea PM. Augmented and virtual reality in anatomical education: a systematic review. In: Rea P, ed. Biomedical Visualisation: Advances in Experimental Medicine and Biology. Springer; 2020:89-101. https://doi. org/10.1007/978-3-030-37639-0 5

16. Aebersold M, Tschannen D, Bathish M. Innovative simulation strategies in education. Nurs Res Pract. 2012;2012:765212. https://doi. org/10.1155/2012/765212

17. Dyer E, Swartzlander BJ, Gugliucci MR. Using virtual reality in medical education to teach empathy. J Med Libr Assoc. 2018;106(4):498-500. https://doi.org/10.5195/jmla.2018.518

18. Khan S. The One World Schoolhouse: Education Reimagined. New York, NY: Grand Central Publishing; 2012. 